\title{
Comparação entre a Prescrição de Intensidade de Treinamento Físico Baseada na Avaliação Ergométrica Convencional e na Ergoespirométrica
}

\author{
Maria Urbana Pinto Brandão Rondon, Cláudia Lúcia de Moraes Forjaz, N ewton N unes, \\ Sandra Lia do Amaral, Antonio Carlos Pereira Barretto, Carlos Eduardo N egrão
}

São Paulo, SP

Objetivo - Comparar os limites inferiores (L.inf.) e superiores (L.sup.) da prescrição de treinamento físico aeróbio determinada pelo teste ergométrico convencional (60-70\% do $\mathrm{VO}_{2}$ máx estimado ou 70-85\% da FCmáx atingida), com a prescrição obtida pelo teste ergoespirométrico [limiar anaeróbio (LA) e ponto de compensação respiratória $(P C R)]$.

Métodos - Realizaram teste ergoespirométrico pro-

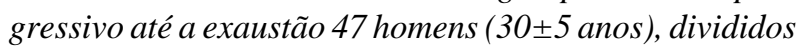
em subgrupos, de acordo com a velocidade da esteira durante o teste (4 ou $5 \mathrm{mph}$ ) e a capacidade física medida [baixa $(B C F)$ e moderada (MCF)].

Resultados - Os L.inf. de prescrição indireta apresentaram valores de $\mathrm{VO}_{2}$ e FC significantemente maiores que os valores de $\mathrm{VO}_{2}$ e FC no LA (4mph $=34,4 \pm 4,5$ vs 19,6 $\pm 4,6$ e $5 \mathrm{mph}=28,9 \pm 2$ vs $18,9 \pm 5,4$, e $B C F=32,0 \pm 4,1$ vs $17,2 \pm 2,8$ e $\mathrm{MCF}=31,6 \pm 4,9 \mathrm{vs} 21,1 \pm 5,7\left(\mathrm{mlO}_{2} \cdot \mathrm{kg}^{-1} \cdot \mathrm{min}^{-1}\right) e$ $(4 \mathrm{mph}=128,9 \pm 7,8$ vs 113,1 $\pm 15,6$ e $5 \mathrm{mph}=130,3 \pm 5,2 \mathrm{vs}$ $114,1 \pm 18,9$, е $B C F=127,6 \pm 7,2$ vs 109,3 $\pm 13,2$ e $M C F=$

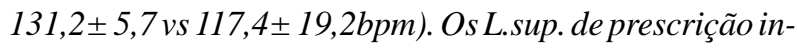
direta no grupo de 4mph e BCF apresentaram valores de $V \mathrm{O}_{2}$ significantemente maiores que os valores medidos no PCR $(40,1 \pm 5,3$ vs $32,2 \pm 4,3$ e $37,4 \pm 4,8$ vs $30,6 \pm 2,5$ $\mathrm{mlO}_{2} \cdot \mathrm{kg}^{-1} \cdot \mathrm{min}^{-1}$, respectivamente), e valores de FC semelhantes aos medidos no PCR.

Conclusão - Os L.inf. da prescrição indireta de treinamento físico superestimam o LA, enquanto os L.sup. parecem adequados somente para indivíduos ativos com $\mathrm{MCF}$.

Palavras-chave: prescrição de exercício, freqüência cardíaca, consumo de oxigênio máximo

\section{Comparison Between Exercise Intensity Prescription Based on a Standard Exercise Test and Cardiopulmonary Exercise Test}

Purpose - To compare the lower (LL) and upper limits (UL) of exercise intensity prescription based on standard exercise test (60-70\% of estimated $\mathrm{VO}_{2}$ max or 70-85\% of HRmax measured) with exercise intensity prescription based on cardiopulmonary exercise test [anaerobic threshold (AT) and respiratory compensation point (RCP)].

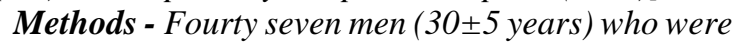
submitted to a progressive cardiopulmonary exercise test until exhaustion were divided in subgroups according to treadmill speed during exercise test ( 4 or $5 \mathrm{mph}$ ) and the physical capacity [lower (LPC) and moderate physical capacity $(M P C)]$.

Results - The LL of the indirect exercise intensity prescription showed $\mathrm{VO}_{2}$ and $\mathrm{HR}$ values significantly higher than $\mathrm{VO}_{2}$ and $\mathrm{HR}$ values measured at $A T(4 \mathrm{mph}=34.4 \pm 4.5$ vs $19.6 \pm 4.6$ and $5 \mathrm{mph}=28.9 \pm 2$ vs $18.9 \pm 5.4$, and $L P C=$ $32.0 \pm 4.1$ vs $17.2 \pm 2.8$ and $M P C=31.6 \pm 4.9$ vs $21.1 \pm$ $\left.5.7 \mathrm{mlO}_{2} \cdot \mathrm{kg}^{-1} \cdot \mathrm{min}^{-1}\right)$ and $(4 \mathrm{mph}=128.9 \pm 7.8 \mathrm{vs} 113.1 \pm 15.6$ and $5 \mathrm{mph}=130.3 \pm 5.2$ vs $114.1 \pm 18.9$, and $L P C=$ $127.6 \pm 7.2$ vs $109.3 \pm 13.2$ and $M P C=131.2 \pm 5.7$ vs $117.4 \pm 19.2 \mathrm{bpm}$ ) The UL of the indirect exercise intensity prescription in 4mph and LPC group showed $V O$, values significantly higher than those measured at RCP $(40.1 \pm 5.3$ vs $32.2 \pm 4.3$ and $37.4 \pm 4.8$ vs $30.6 \pm 2.5 \mathrm{mlO}_{2} \cdot \mathrm{kg}^{-1} \cdot \mathrm{min}^{-1}$, respectively), but similar HR values to those obtained at $R C P$.

Conclusion - The LL of prescription based on standard exercise test overestimate the AT, whereas the UL seem adequate only for subjects with moderate physical capacity.

Key-words: exercise prescription, heart rate, maximal oxygen uptake

Arq Bras Cardiol, volume 70 (nº 3), 159-166, 1998

Instituto do Coração do Hospital das Clínicas - FMUSP

Correspondência: Maria U. P. Brandão Rondon - Incor - Serviço de Condicionamento

Físico - Av. Dr. Enéas C. Aguiar, 44 - 05403-000 - São Paulo, SP

Recebido para publicação em 19/11/97

Aceito em 7/1/98
Embora pareçam existir poucas dúvidas quanto a melhora na qualidade de vida e, sobretudo, na condição de saúde alcançada através de um programa de condicionamento físico, esses benefícios dependem de uma prescrição 
de exercício adequada, no que diz respeito a sua intensidade, duração, freqüência e modalidade. Dentre esses fatores, a intensidade do exercício parece ter um papel de destaque no resultado final alcançado ${ }^{1}$.

Nesse sentido, a ergoespirometria computadorizada veio proporcionar um avanço importante para o desenvolvimento de um programa de condicionamento físico, uma vez que possibilita avaliar, de maneira precisa, a capacidade cardiorrespiratória e metabólica, através da medida direta do consumo de oxigênio máximo e da determinação dos limiares ventilatórios [limiar anaeróbio (LA) e ponto de compensação respiratória]. Esses limiares fornecem, de forma nãoinvasiva e com grande precisão, as intensidades de exercício em que predominam o metabolismo aeróbio e anaeróbio ${ }^{2,3}$, possibilitando uma prescrição adequada e individualizada da intensidade do condicionamento físico. Em nossa experiência, essa prescrição é atingida com o exercício físico realizado de maneira regular, numa faixa de intensidade que varia entre o LA e o ponto de compensação respiratória.

$\mathrm{O}$ acesso pouco freqüente e o alto custo da avaliação ergoespirométrica, no entanto, fazem com que os testes de esforço convencionais sejam mais utilizados que a ergoespirometria na avaliação da capacidade funcional. Se por um lado, os testes convencionais avaliam adequadamente as respostas cardiovasculares durante o exercício, por outro, utilizam-se de métodos indiretos para determinar o consumo de oxigênio máximo e, conseqüentemente, prescrever a intensidade de exercício a ser desenvolvida no programa de condicionamento físico aeróbio ${ }^{4,5}$. Esta intensidade prescrita indiretamente, normalmente, baseia-se na recomendação do American College of Sports Medicine (ACSM) ${ }^{4}$, que preconiza para indivíduos não-idosos saudáveis, uma intensidade de exercício entre 60 e $70 \%$ do consumo de oxigênio máximo estimado $\left(\mathrm{VO}_{2}\right.$ máxE) ou entre 70 e $85 \%$ da freqüência cardíaca máxima medida (FCmáxM) no teste de esforço (TE).

A estimativa do consumo de oxigênio máximo, na maioria das vezes, leva em consideração a potência máxima atingida no TE. Acontece, porém, que nem sempre o TE é interrompido em carga máxima de exercício, o que acaba distorcendo ainda mais a prescrição de intensidade de exercício físico.

Pouco se conhece a respeito da relação existente entre a intensidade de exercício prescrita, de forma indireta, e a intensidade estabelecida pelos limiares ventilatórios, ou seja, o quanto os limites inferiores de prescrição $(60 \%$ do $\mathrm{VO}_{2}$ máxE ou $70 \%$ da FCmáxM) e os superiores (70\% do $\mathrm{VO}_{2}$ máxE e $85 \%$ da FCmáxM) propostos pelo $\mathrm{ACSM}^{4}$ são efetivos para representar o início da intensificação do metabolismo anaeróbio (LA) e o início da fase de descompensação da acidose metabólica (ponto de compensação respiratória), respectivamente.

Nosso objetivo foi comparar os limites inferior e superior da faixa de prescrição de intensidade de exercício físico prescrito pelo $\mathrm{ACSM}^{4}$, calculados a partir do TE convencional, com a prescrição relativa ao LA e ponto de compensação respiratória obtidos no teste ergoespirométrico.

\section{Métodos}

Foram avaliados 47 homens saudáveis, voluntários, do sexo masculino, com idade média de 29,7 $\pm 5,0$ anos, peso médio de $76,5 \pm 9,7 \mathrm{~kg}$ e estatura média de $175 \pm 5 \mathrm{~cm}$. O consumo de oxigênio máximo medido, diretamente, foi de $42,1 \pm$ $3,5 \mathrm{mlO}_{2} \cdot \mathrm{kg}^{-1} \cdot \mathrm{min}^{-1}$.

Foi realizado inicialmente um eletrocardiograma(ECG) de repouso em eletrocardiógrafo computadorizado (TecnologiaEletrônica Brasileira-TEB, SM310), com o registro das 12 derivações padrão. Em seguida, os indivíduos realizaram um teste ergométrico na esteira rolante (Quinton), seguindo-se o protocolo de Balke modificado com velocidade cruzeiro de 4 ou 5 mph e incrementos de $2 \%$ na inclinação a cada minuto até a exaustão. A velocidade da esteira foi selecionada, a partir da capacidade física individual relatada por cada indivíduo. Nenhum dos voluntários apresentou problemas cardiovasculares e todos os testes foram interrompidos por cansaço físico intenso.

Durante todo o TE, o indivíduo foi continuamente monitorizado através do ECG, utilizando-se três derivações simultâneas (MC5, D2M, V2M). A freqüência cardíaca(FC) foi registrada ao final do $2^{\circ}$ minuto de repouso em pé na esteira, ao final de cada minuto de exercício e no $1^{\circ}, 2^{\circ}, 4^{\circ}$ e $6^{\circ}$ minutos de recuperação. A pressão arterial foi aferida pelo método auscultatório, utilizando-se esfigmomanômetro de coluna de mercúrio, no final do repouso, a cada 2 min durante o exercício e no $1^{\circ}, 2^{\circ}, 4^{\circ}$ e $6^{\circ}$ minutos de recuperação.

Durante o TE, o ar expirado foi coletado e analisado a cada ciclo respiratório pelo analisador de gases computadorizado (Medical Graphics Corporation - MGC, mod CAD/Net 2001). A ventilação pulmonar foi medida por um pneumotacógrafo e as concentrações de oxigênio $\left(\mathrm{O}_{2}\right)$ e dióxido de carbono $\left(\mathrm{CO}_{2}\right)$ foram aferidas, respectivamente, através de célula de zircônio e ondas infravermelho. A partir das análises da ventilação e das concentrações de $\mathrm{O}_{2} \mathrm{e} \mathrm{CO}_{2}$ expirados foram calculados o consumo de oxigênio $\left(\mathrm{VO}_{2}\right) \mathrm{e}$ a produção de dióxido de carbono $\left(\mathrm{VCO}_{2}\right)$. $\mathrm{O}$ consumo de oxigênio máximo medido $\left(\mathrm{VO}_{2}\right.$ máxM) diretamente no teste foi considerado como o valor obtido no pico do exercício, quando o indivíduo encontrava-se em exaustão, calculado em médias de 60s. A FC, obtida pelo eletrocardiógrafo, foi

Tabela I - Características físicas da amostra completa e dos grupos separados pela condição física relatada (protocolo de 4 ou $5 \mathrm{mph}$ ) e medida diretamente no teste ergoespirométrico (BCF e MCF)

\begin{tabular}{|c|c|c|c|c|c|}
\hline \multirow[t]{2}{*}{ Variável } & \multirow{2}{*}{$\begin{array}{l}\text { Amostra } \\
\text { completa }\end{array}$} & \multicolumn{2}{|c|}{$\begin{array}{l}\text { Condição física } \\
\text { relatada }\end{array}$} & \multicolumn{2}{|c|}{$\begin{array}{c}\text { Condição física } \\
\text { medida }\end{array}$} \\
\hline & & $\begin{array}{l}4 \mathrm{mph} \\
(\mathrm{n}=25)\end{array}$ & $\begin{array}{c}5 \mathrm{mph} \\
(\mathrm{n}=22)\end{array}$ & $\begin{array}{c}\mathrm{BCF} \\
(\mathrm{n}=22)\end{array}$ & $\begin{array}{r}\text { MCF } \\
(n=25)\end{array}$ \\
\hline Idade (anos) & $30 \pm 5$ & $30 \pm 5$ & $29 \pm 5$ & $31 \pm 5$ & $28 \pm 5$ \\
\hline Peso (kg) & $77 \pm 10$ & $76 \pm 11$ & $77 \pm 8$ & $78 \pm 11$ & $75 \pm 9$ \\
\hline Estatura $(\mathrm{cm})$ & $175 \pm 5$ & $176 \pm 5$ & $174 \pm 6$ & $174 \pm 5$ & $176 \pm 6$ \\
\hline $\operatorname{IMC}\left(\mathrm{kg} / \mathrm{m}^{2}\right)$ & $25,1 \pm 3,1$ & $24,7 \pm 3,5$ & $25,5 \pm 2,5$ & $25,7 \pm 3,4$ & $24,5 \pm 2,8$ \\
\hline
\end{tabular}

BCF- baixa capacidade física; MCF- moderada capacidade física; IMC- índice de massa corporal. 


\begin{tabular}{|c|c|c|c|c|c|}
\hline \multirow[t]{2}{*}{ Variável } & \multirow[t]{2}{*}{ Amostra completa } & \multicolumn{2}{|c|}{ Condição física relatada } & \multicolumn{2}{|c|}{ Condição física medida } \\
\hline & & $4 \mathrm{mph}$ & $5 \mathrm{mph}$ & $\mathrm{BCF}$ & MCF \\
\hline $\mathrm{VO}_{2} \operatorname{máxM}\left(\mathrm{mlO}_{2} \cdot \mathrm{kg}^{-1} \cdot \mathrm{min}^{-1}\right)$ & $42,1 \pm 3,5^{+}$ & $40,7 \pm 3,4^{*,+}$ & $43,7 \pm 3,1^{+}$ & $39,1 \pm 2,3^{\#,+}$ & $44,8 \pm 1,8^{+}$ \\
\hline $\mathrm{VO}_{2} \operatorname{máxE}\left(\mathrm{mlO}_{2} \cdot \mathrm{kg}^{-1} \cdot \mathrm{min}^{-1}\right)$ & $53,0 \pm 7,5$ & $57,3 \pm 7,5^{*}$ & $48,1 \pm 3,3$ & $53,4 \pm 6,8^{\#}$ & $52,7 \pm 8,2$ \\
\hline FCmáxM(bpm) & $185 \pm 9^{\circ}$ & $184 \pm 11^{\circ}$ & $186 \pm 7^{\circ}$ & $182 \pm 10^{\circ}$ & $188 \pm 8^{\circ}$ \\
\hline FCmáxP(bpm) & $190 \pm 5$ & $189 \pm 5$ & $191 \pm 5$ & $189 \pm 5$ & $192 \pm 5$ \\
\hline$\%$ do $\mathrm{VO}_{2}$ máxM no LA & $46 \pm 11$ & $48 \pm 10$ & $43 \pm 11$ & $44 \pm 8$ & $47 \pm 13$ \\
\hline$\%$ do $\mathrm{VO}_{2}$ máxM no PCR & $78 \pm 9$ & $79 \pm 9$ & $76 \pm 8$ & $79 \pm 8$ & $77 \pm 9$ \\
\hline \% da FCmáxM no LA & $61 \pm 8$ & $61 \pm 7$ & $61 \pm 9$ & $60 \pm 7$ & $63 \pm 9$ \\
\hline \% da FCmáxM no PCR & $84 \pm 7$ & $85 \pm 7$ & $84 \pm 8$ & $84 \pm 6$ & $84 \pm 8$ \\
\hline \multicolumn{6}{|c|}{ 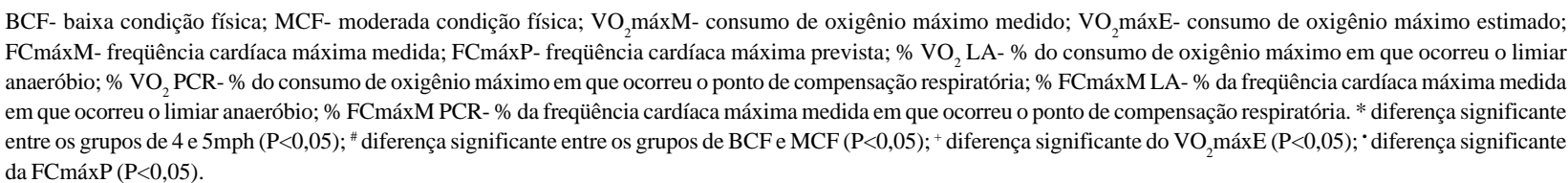 } \\
\hline
\end{tabular}

transmitida como sinal analógico ao analisador de gases computadorizado que a calculou em médias de 60s. Da mesma forma, a FC obtida no pico do exercício foi considerada como a FCmáxM no teste.

O consumo de oxigênio máximo estimado ( $\mathrm{VO}_{2}$ máxE) foi calculado indiretamente a partir da velocidade e da inclinação máxima atingida no TE, utilizando-se as fórmulas propostas pelo ACSM $^{5}$ : teste com protocolo de caminhada (4mph): $\mathrm{VO}_{2}\left(\mathrm{mlO}_{2} \cdot \mathrm{kg}^{-1} \cdot \mathrm{min}^{-1}\right)=\left[\operatorname{velocidade}\left(\mathrm{ml} \cdot \mathrm{min}^{-1}\right) \times 0,1\right]$ $+\left[\right.$ velocidade $\left(\mathrm{ml} \cdot \mathrm{min}^{-1}\right) \mathrm{x} \%$ inclinaçãox 1,8$]+3,5 \mathrm{mlO}_{2} \cdot \mathrm{kg}^{-1} \cdot \mathrm{min}^{-1}$. Teste com protocolo de corrida (5mph): $\mathrm{VO}_{2}\left(\mathrm{mlO}_{2} \cdot \mathrm{kg}^{-1} \cdot \mathrm{min}^{-1}\right)$ $=\left[\operatorname{velocidade}\left(\mathrm{ml} \cdot \mathrm{min}^{-1}\right) \times 0,2\right]+\left[\operatorname{velocidade}\left(\mathrm{ml} \cdot \mathrm{min}^{-1}\right) \times \%\right.$ inclinação x $1,8 \times 0,5]+3,5 \mathrm{mlO}_{2} \cdot \mathrm{kg}^{-1} \cdot \mathrm{min}^{-1}$

A freqüência cardíaca máxima prevista $(\mathrm{FCmáxP})$ para a idade foi calculada pela fórmula: $\mathrm{FCmáxP}=220$ - idade (anos) ${ }^{6}$.

Com os dados da ergoespirometria foram determinados também o LA e o ponto de compensação respiratória (PCR). OLA foi considerado no minuto de exercício em que se observou um incremento não linear da razão de troca respiratória (RER) e os menores valores do equivalente ventilatório de oxigênio $\left(\mathrm{VE} / \mathrm{VO}_{2}\right)$ e da pressão parcial final de oxigênio $\left(\mathrm{PetO}_{2}\right)$, isto é, antes do início do aumento progressivo dessas respostas. O PCR foi considerado no minuto de exercício em que se observou o menor valor do equivalente ventilatório de dióxido de carbono $\left(\mathrm{VE} / \mathrm{VCO}_{2}\right)$, ou seja, antes do início do seu aumento progressivo, e o maior valor da pressão parcial final de dióxido de carbono $\left(\mathrm{PetCO}_{2}\right)$.

Para a prescrição de intensidade de treinamento físico aeróbio, baseada no teste ergoespirométrico, foram utilizados como limite inferior de prescrição, os valores de $\mathrm{VO}_{2} \mathrm{e}$ FC medidos no LA e, como limite superior, os valores de $\mathrm{VO}_{2}$ e FC medidos no PCR.

Para a prescrição indireta da intensidade de treinamento físico aeróbio, baseada no teste ergométrico, foram utilizados os seguintes procedimentos: a) $60 \%$ do $\mathrm{VO}_{2}$ máxE e $70 \%$ da FCmáxM, como limite inferior da intensidade de treinamento e $70 \%$ do $\mathrm{VO}_{2}$ máxEe $85 \%$ daFCmáxM como limite superior da intensidade de treinamento e, b) $70 \%$ da FCmáxP para a idade como limite inferior da intensidade de treinamento e $85 \%$ da FCmáxP para a idade como limite superior da intensidade de treinamento.

Para a comparação das faixas de intensidade de treinamento físico prescritas pelo método direto (ergoespirometria) e pelo método indireto (estimado) foram comparados os limites inferiores e superiores dessas prescrições, tanto em relação à FC quanto ao $\mathrm{VO}_{2}$. Essas comparações foram realizadas pelo teste-t de Student para amostras repetidas e o nível de $\mathrm{P}<0,05$ foi aceito como significante. Os dados serão apresentados com média \pm desvio-padrão. Essa análise, inicialmente, foi realizada com todos os indivíduos estudados (análise experimental 1)e, posteriormente, com os indivíduos divididos em dois subgrupos, segundo a capacidade física individual relatada ou medida. Essa divisão foi realizada da seguinte maneira: a) indivíduos que realizaram TE com velocidade fixada em $4 \mathrm{mph}$ e indivíduos que realizaram TE com velocidade fixada em $5 \mathrm{mph}$. Este critério de classificação baseou-se na capacidade física máxima relatada por cada indivíduo estudado (análise experimental 2); b) indivíduos que possuíam baixa capacidade física máxima (BCF) e indivíduos que possuíam moderada capacidade física máxima (MCF). Essa classificação baseou-se no resultado do teste ergoespirométrico em que se mediu diretamente o consumo de oxigênio máximo e foi calculada a média aritmética do $\mathrm{VO}_{2}$ máxM de todos os indivíduos estudados. Os indivíduos que tiveram seu $\mathrm{VO}_{2}$ máxM abaixo da média, foram alocados no grupo BCF e os indivíduos que tiveram seu $\mathrm{VO}_{2}$ máxM acima da média, foram alocados no grupo MCF (análise experimental 3).

As características físicas e a capacidade funcional dos grupos estão apresentadas na tabela I.

\section{Resultados}

Análise experimental 1 - Respostas cardiorrespiratórias e metabólicas máximas de todos indivíduos estudados. Analisando-se todos os indivíduos estudados ( $\mathrm{n}=47)$, 


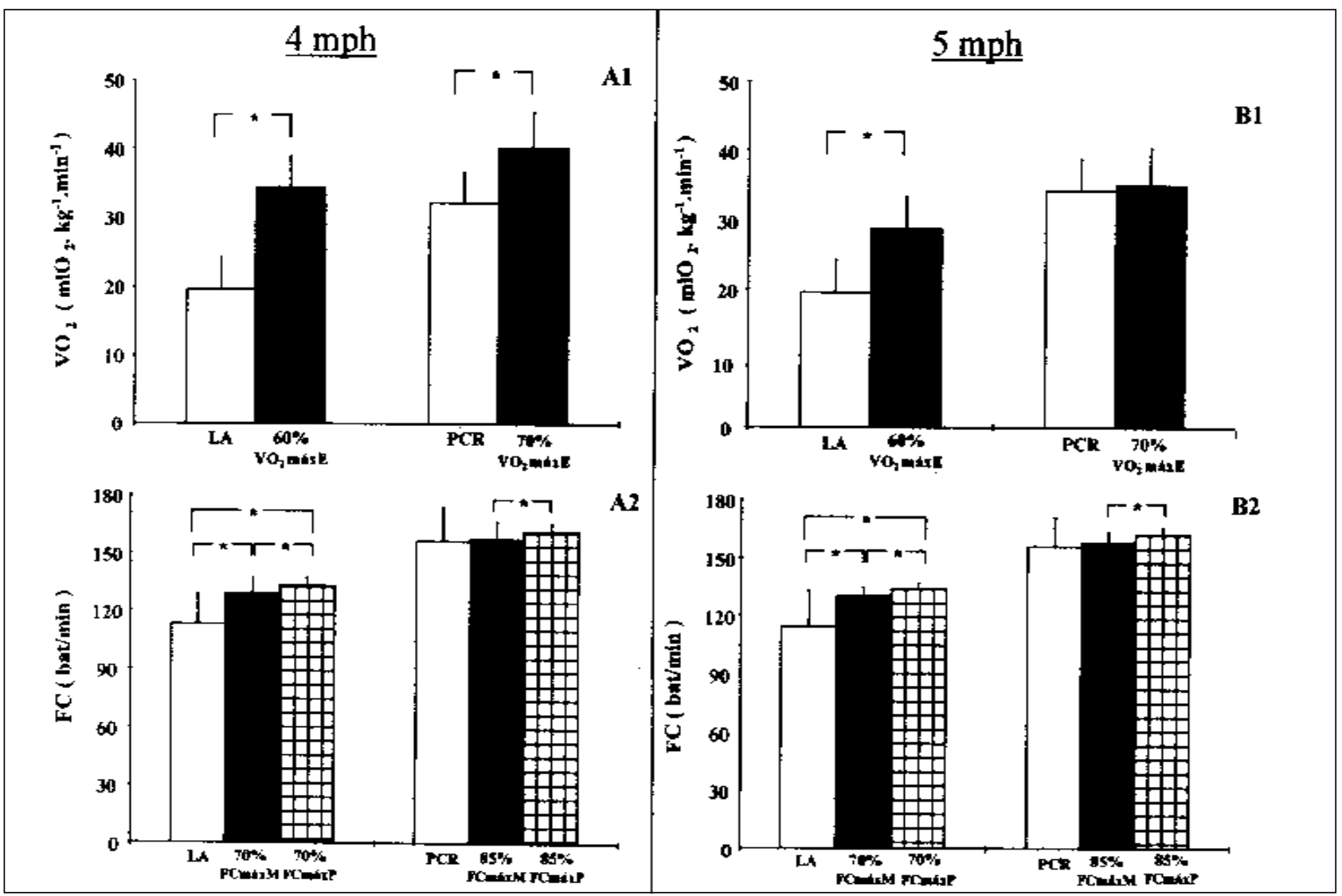

Fig. 1 - Comparação dos limites inferiores e superiores de uma prescrição de um programa de condicionamento físico baseado nos limiares ventilatórios (barras brancas), nas porcentagens do consumo de oxigênio máximo e freqüência cardíaca máxima estimadas a partir do teste de esforço (barras pretas) e nas porcentagens da FCmáxP para a idade (barras quadriculadas). Os painéis A1 e B1 mostram o consumo de oxigênio e os painéis A2 e B2 a frequiência cardíaca em indivíduos que realizaram teste de esforço com velocidade fixa de $4 \mathrm{mph}$ (painéis A1 e A2) e de $5 \mathrm{mph}$ (painéis B1 e B2). LA- limiar anaeróbio; PCR-ponto de compensação respiratória; $\mathrm{VO}_{2}$ - consumo de oxigênio; VO máxE- consumo de oxigênio máximo estimado; FC- freqüência cardíaca; FCmáxM- freqüência cardíaca máxima medida; FCmáxP- freqüência cardíaca máxima prevista para a idade. * diferença significante (P<0,05).

verificou-se que o $\mathrm{VO}_{2}$ máxE calculado por fórmula matemática, foi significantemente maior que o $\mathrm{VO}_{2}$ máxM através da ergoespirometria $\left(53 \pm 7,5\right.$ vs $42,1 \pm 3,5 \mathrm{mlO} 2 \cdot \mathrm{kg}^{-1} \cdot \mathrm{min}^{-1}$, $\mathrm{P}<0,05$, tab. II). A FCmáxP também foi significantemente

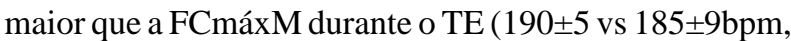
$\mathrm{P}<0,05)$. Esses resultados evidenciam que: 1) o $\mathrm{VO}$ máxE através de fórmulas foi em média $21 \%$ maior que o $\mathrm{VO}_{2}$ máxM diretamente no pico do exercício através da ergoespirometria e, 2) a FCmáxP foi em média 3\% maior que aquela medida diretamente no pico do exercício.

Limite inferior de prescrição de intensidade de exercício para um programa de condicionamento físico de todos indivíduos estudados - O LA foi atingido em $46 \pm 11 \%$ do $\mathrm{VO}_{2}$ máxM e $61 \pm 8 \%$ daFCmáxM(tab. II). O consumo de oxigênio calculado em $60 \%$ do $\mathrm{VO}_{2}$ máxE (limite inferior estimado de intensidade de exercício) foi significantemente maior que o consumo de oxigênio medido diretamente no LA $\left(31,8 \pm 4,5\right.$ vs $\left.19,2 \pm 4,9 \mathrm{mlO}_{2} \cdot \mathrm{kg}^{-1} \cdot \mathrm{min}^{-1}, \mathrm{P}<0,05\right)$. Da mesmaforma, a FC calculada em 70\% da FCmáxM (limite inferior estimado de intensidade de exercício) também foi significantemente maior que a FC medida no momento em que ocorreu o LA, determinado pelo método ergoespirométrico (130 \pm 7 vs $114 \pm 17$ bpm, $\mathrm{P}<0,05)$. E, a FC calculada em $70 \%$ da

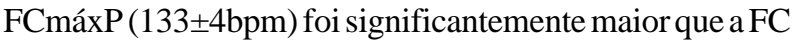

medida no LA e aquela calculada em $70 \%$ da FCmáxM. Esses resultados evidenciam que: 1) o consumo de oxigênio calculado em $60 \%$ do $\mathrm{VO}_{2}$ máxE e preconizado como limite inferior estimado de intensidade de exercício para um programa de condicionamento físico foi em média $40 \%$ maior que o consumo de oxigênio medido no LA e, 2) a FC calculada em $70 \%$ daFCmáxM ou 70\% daFCmáxP foram em média 12 e $14 \%$ maiores que a FC medida no LA.

Limite superior de prescrição de intensidade de exercício para um programa de condicionamento físico de todos indivíduos estudados - O ponto de compensação respiratória foi atingido em $78 \pm 9 \%$ do $\mathrm{VO}_{2}$ máxM e $84 \pm 7 \%$ da FC máxM. O consumo de oxigênio calculado em $70 \%$ do $\mathrm{VO}_{2}$ máxE foi significantemente maior que o consumo de oxigênio medido diretamente no momento em que ocorreu o ponto de compensação respiratória $(37,1 \pm 5,3$ vs $32,7 \pm$ $\left.4,1 \mathrm{mlO} \cdot \mathrm{kg}^{-1} \cdot \mathrm{min}^{-1}, \mathrm{P}<0,05\right)$. A FC calculada em $85 \%$ da FCmáxM foi semelhante à FC medida no ponto de compensação respiratória ( $157 \pm 8$ vs $156 \pm 17 \mathrm{bpm})$. No entanto, a prescrição calculada em $85 \%$ da FCmáxP $(162 \pm 8 \mathrm{bpm})$ foi significantemente maior que a FC medida no ponto de compensação respiratória e aquela calculada em $85 \%$ da FCmáxM. Esses resultados evidenciam que: 1) o consumo de oxigênio calculado em $70 \%$ do $\mathrm{VO}_{2}$ máxE e preconizado 


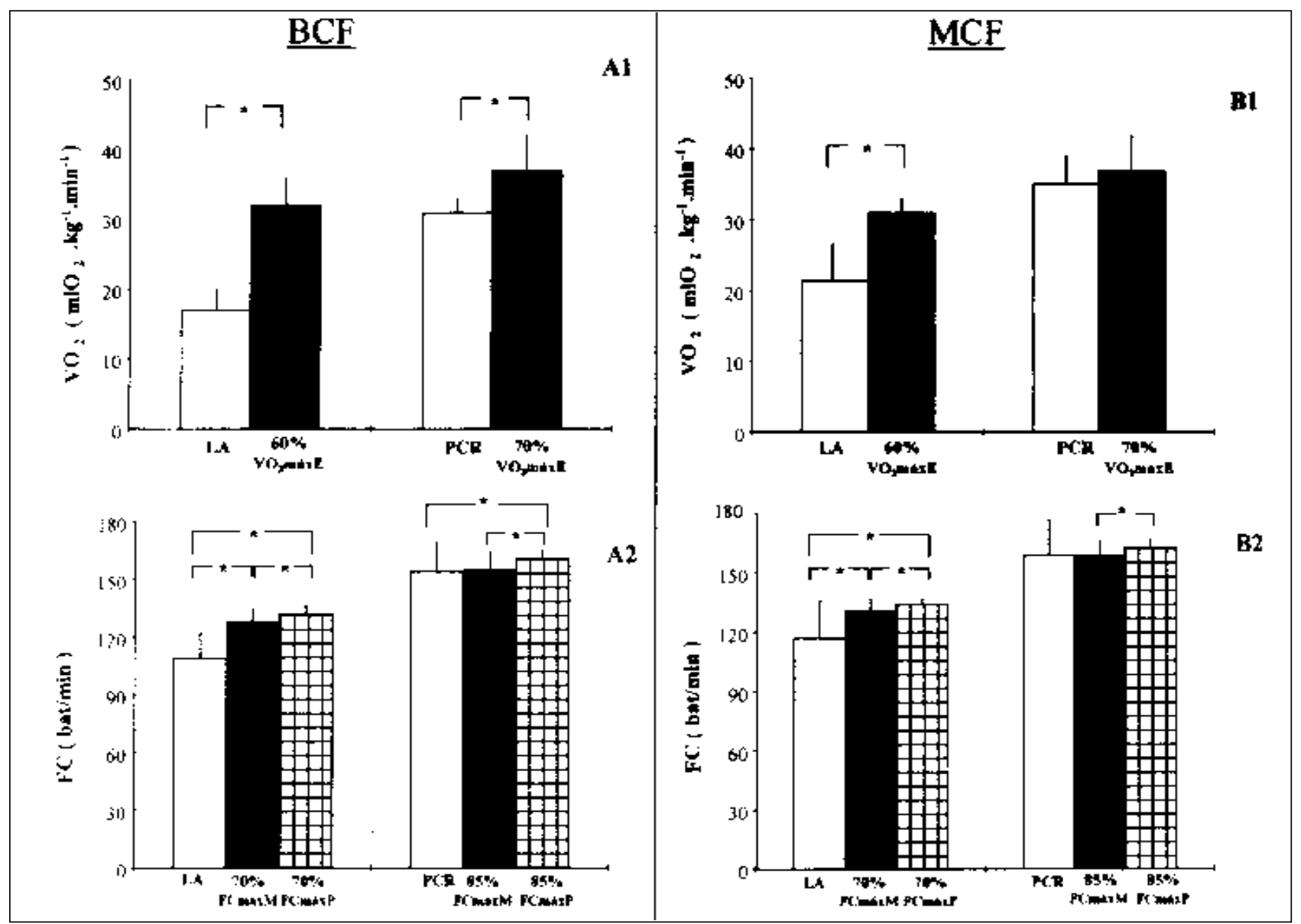

Fig. 2 - Comparação dos limites inferiores e superiores de uma prescrição de um programa de condicionamento físico baseado nos limiares ventilatórios (barras brancas), nas porcentagens do consumo de oxigênio máximo e frequiência cardíaca máxima estimadas a partir do teste de esforço (barras pretas) e nas porcentagens da freqüência cardíaca máxima prevista para a idade (barras quadriculadas). Os painéis $\mathrm{A} 1$ e B1 mostram o consumo de oxigênio e os painéis A2 e B2 a frequiência cardíaca em indivíduos que apresentavam VO máx inferior (baixa condição física - BCF) e superior (moderada condição física - MCF) à média do grupo. LA- limiar anaeróbio; PCR- ponto de compensação respiratória; VO2- consumo de oxigênio; VO2máxE- consumo de oxigênio máximo estimado; FC- freqüência cardíaca; FCmáxM-freqüência cardíaca máxima medida; FCmáxP- freqüência cardíaca máxima prevista para a idade. $*$ diferença significante $(\mathrm{P}<0,05)$.

como limite superior de intensidade de exercício para um programa de condicionamento foi em média, $12 \%$ maior que o consumo de oxigênio medido no ponto de compensação respiratóriae 2) aFC calculada em $85 \%$ da FCmáxM foi semelhante aquela medida no ponto de compensação respiratória e que essas duas freqüências foram $4 \%$ menores que $85 \%$ daFCmáxP.

Análise experimental 2 (protocolo de 4 ou $5 \mathrm{mph}$ ) respostas cardiorrespiratórias e metabólicas máximas baseadas no TE com velocidade de 4 ou $5 \mathrm{mph}$ - $\mathrm{O} \mathrm{VO}$ máxM foi significantemente maior no grupo que realizou o TE com a velocidade da esteira em $5 \mathrm{mph}$ que no grupo que realizou o testeem $4 \mathrm{mph}$ (tab. II). $\mathrm{OVO}_{2}$ máxEfoi significantemente maior que o $\mathrm{VO}_{2}$ máxM tanto no grupo que realizou o teste com o protocolo em $4 \mathrm{mph}\left(57,3 \pm 7,5\right.$ vs $\left.40,7 \pm 3,4 \mathrm{mlO} \cdot \mathrm{kg}^{-1} \cdot \mathrm{min}^{-1}\right)$ quanto no grupo que realizou o protocolo em $5 \mathrm{mph}$ $\left(48,1 \pm 3,3\right.$ vs $\left.43,7 \pm 3,1 \mathrm{mlO}_{2} \cdot \mathrm{kg}^{-1} \cdot \mathrm{min}^{-1}\right)$. De maneira semelhante, a FCmáxP foi significantemente maior que a FCmáxM nos dois protocolos de teste $(4 \mathrm{mph}=189 \pm 5$ vs $184 \pm 11 \mathrm{bpm}$; $5 \mathrm{mph}=191 \pm 5$ vs $186 \pm 7 \mathrm{bpm}, \mathrm{P}<0,05$, tab. II). Esses resultados evidenciam que: 1 ) o $\mathrm{VO}_{2}$ máxM do subgrupo que reali- zou o teste com velocidade de $5 \mathrm{mph}$ foi em média $3 \%$ maior que aqueles que realizaram o teste em $4 \mathrm{mph}$; 2) o $\mathrm{VO}_{2}$ máxE foi 29 e $9 \%$ maiores que o $\mathrm{VO}_{2}$ máxM nos indivíduos que realizaram o teste em 4 e 5mph, respectivamente e, 3) aFCmáxP foi $3 \%$ maior que a FCmáxM no pico do exercício, tanto nos indivíduos que realizaram o teste em 4 quanto em $5 \mathrm{mph}$.

Limite inferior de prescrição de intensidade de exercício para um programa de condicionamento físico baseado no TE com velocidade de 4 ou $5 \mathrm{mph}$ - O LA foi alcançado em $48 \pm 10 \%$ do $\mathrm{VO}_{2}$ máxM e $61 \pm 7 \%$ daFCmáxM no protocolo de 4mph e em $43 \pm 11 \%$ do $\mathrm{VO}_{2}$ máxM e $61 \pm 9 \%$ da FCmáxM no protocolo de $5 \mathrm{mph}$. A prescrição indireta calculada em $60 \%$ do $\mathrm{VO}_{2}$ máxE foi significantemente maior que o consumo de oxigênio medido no LA, tanto no teste em $4 \mathrm{mph}$ como no teste em 5mph (Fig. 1, A1 e B1). A FC prescrita em 70\% da FCmáxM foi significantemente maior que a FC medida no LA em ambos, o protocolo em $4 \mathrm{mph}$ e em $5 \mathrm{mph}$ (fig. 1, A2 e B2). Além disso, a prescrição calculada em $70 \%$ da FCmáxP foi significantemente maior que a FC medida no LA e aquela calculada em $70 \%$ da FCmáxM, nos dois protocolos de testes estudados, isto é, 4 e 5mph (fig. 1, A2 e B2 ). Esses 
resultados evidenciam que: 1) o consumo de oxigênio calculado em $60 \%$ do $\mathrm{VO}_{2}$ máxE e preconizado como limite inferior estimado de intensidade de exercício para um programa de condicionamento físico foi em média 43 e $35 \%$ maior que o consumo de oxigênio medido no LA, no TE realizado em $4 \mathrm{e}$ $5 \mathrm{mph}$, respectivamente; 2 ) a FC calculada em $70 \%$ da FCmáxM ou 70\% daFCmáxP foram em média 12e 15\% maiores que a FC medida no LA tanto no TE realizado em $4 \mathrm{mph}$ como no teste realizado em $5 \mathrm{mph}$; 3) a FC calculada em $70 \%$ da FCmáxP foi 3 e $2 \%$ maior que os valores de FC em $70 \%$ da FCmáxM nos testes realizados em 4 e $5 \mathrm{mph}$, respectivamente.

Limite superior de prescrição de intensidade de exercício para um programa de condicionamento físico baseado no TE com velocidade de 4 ou $5 \mathrm{mph}$ - O ponto de compensação respiratória foi atingido em $79 \pm 9 \%$ do $\mathrm{VO}_{2}$ máxM e em $85 \pm 7 \%$ da FCmáxM no protocolo de $4 \mathrm{mph}$ (tab. II) e em $76 \pm 8 \%$ do $\mathrm{VO}_{2}$ máxM e $84 \pm 8 \%$ daFCmáxM no protocolo de $5 \mathrm{mph}$ (tab. II). O consumo de oxigênio em $70 \%$ do $\mathrm{VO}_{2}$ máxE foi significantemente maior que o consumo de oxigênio no ponto de compensação respiratória no protocolo de $4 \mathrm{mph}$, mas não no protocolo de 5mph (fig. 1, A1 e B1). A prescrição de intensidade de exercício calculada em $85 \%$ da FCmáxM não foi estatisticamente diferente da FC medida no ponto de compensação respiratória em nenhum dos protocolos (fig. 1, A2 e B2). No entanto, quando a prescrição foi calculada em $85 \%$ da FCmáxP, verificou-se que ela era significantemente maior que a FC em $85 \%$ da FCmáxM. Esses resultados foram observados tanto no protocolo de $4 \mathrm{mph}$ quanto no protocolo de $5 \mathrm{mph}$ (fig. 1, A2 e B2). Esses resultados evidenciam que: 1) o consumo de oxigênio calculado em $70 \%$ do $\mathrm{VO}_{2}$ máxEe preconizado como limite superior de intensidade de exercício para um programa de condicionamento físico foi em média $20 \%$ maior que o consumo de oxigênio medido no ponto de compensação respiratória no TE realizado na velocidade de $4 \mathrm{mph}$, mas não foi diferente no teste realizado em $5 \mathrm{mph}$; 2) a FC calculada em $85 \%$ da FCmáxM foi semelhante aquela medida no ponto de compensação respiratória no protocolo de teste realizado em $4 \mathrm{e}$ em 5mph; 3) aFC calculada em $85 \%$ daFCmáxP foi em média $3 \%$ maior que os valores de FC em $85 \%$ da FCmáxM nos testes realizados em 4 e $5 \mathrm{mph}$.

Análise experimental 3: respostas cardiorrespiratórias e metabólicas máximas em indivíduos com BCF e MCF - O $\mathrm{VO}_{2}$ máxM foi significantemente maior no grupo de $\mathrm{MCF}$ que no grupo de $\mathrm{BCF}$ (tab. II). $\mathrm{O} \mathrm{VO}_{2}$ máxE foi significantemente maior do que o $\mathrm{VO}_{2}$ máxM tanto no grupo $\mathrm{BCF}(53,4 \pm 6,8$ vs $\left.39,1 \pm 2,3 \mathrm{mlO}_{2} \cdot \mathrm{kg}^{-1} \cdot \mathrm{min}^{-1}\right)$ quanto no $\operatorname{MCF}(52,7 \pm 8,2$ vs $44,8 \pm 1,8 \mathrm{mlO}_{2} \cdot \mathrm{kg}^{-1} \cdot \mathrm{min}^{-1}, \mathrm{tab}$. II). A FCmáxP foi significantemente maior que FCmáxM $(\mathrm{BCF}=189 \pm 5$ vs $182 \pm$ $10 \mathrm{bpm} ; \mathrm{MCF}=192 \pm 5$ vs $188 \pm 8 \mathrm{bpm}, \mathrm{P}<0,05)$. Esses resultados evidenciam que: 1 ) o $\mathrm{VO}_{2}$ máxM do subgrupo com $\mathrm{MCF}$ foi em média $13 \%$ maior que o subgrupo com BCF; 2) o $\mathrm{VO}_{2}$ máxE foi em média 27 e $15 \%$ maior que o $\mathrm{VO}_{2}$ máxM nos indivíduos com BCFe MCF, respectivamente; 3) a FCmáxP foi 4 e $2 \%$ maior que a FCmáxM no pico do exercício, no subgrupo de BCF e MCF, respectivamente.

Limite inferior de prescrição de intensidade de exercí- cio para um programa de condicionamento físico em indivíduos com baixa e MCF - O LA foi alcançado em $44 \pm 8 \%$ do $\mathrm{VO}_{2}$ máxMe $60 \pm 7 \%$ daFCmáxM no grupo BCFeem $47 \pm 13 \%$ do $\mathrm{VO}_{2}$ máxMe $63 \pm 9 \%$ da FCmáxM no grupo MCF (tab. II). A prescrição indireta de exercício calculada em $60 \%$ do $\mathrm{VO}_{2}$ máxE e em $70 \%$ da FCmáxM foram significantemente maiores que o consumo de oxigênio (fig. 2, A1 e B1) e a FC (fig. 2, A2 e B2) medidos no LA nos subgrupos BCF e MCF, respectivamente. Além disso, a prescrição calculada em $70 \%$ daFCmáxP foi significantemente maior que a FC medida no momento em que ocorreu oLA e maior que a FC calculada pela FCmáxM (fig. 2, A2 e B2) nos subgrupos BCF e MCF, respectivamente. Esses resultados evidenciam que: 1) o consumo de oxigênio calculado em $60 \%$ do $\mathrm{VO}_{2}$ máxE e preconizado como limite inferior estimado de intensidade de exercício para um programa de condicionamento físico foi em média 46 e $33 \%$ maior que o consumo de oxigênio medido no LA, nos subgrupos BCF e MCF, respectivamente; 2) aFC calculadaem 70\% daFCmáxM ou 70\% daFCmáxPforam em média 14 e $17 \%$ maiores que a FC medida no LA no subgrupo de BCF e foram em média 11 e $13 \%$ maiores que a FC medida no LA no subgrupo de MCF; 3) a FC calculada em $70 \%$ da FCmáxP foi em média 4 e $2 \%$ maior que os valores de FC em 70\% da FCmáxM nos subgrupos BCF e MCF, respectivamente.

Limite superior de prescrição de intensidade de exercício para um programa de condicionamento físico em indivíduos com baixa e MCF - O ponto de compensação respiratória foi atingido em $79 \pm 8 \%$ do $\mathrm{VO}_{2}$ máxM e em $84 \pm 6 \%$ da FCmáxM no grupo BCF (tab. II) e em 77士9\% do $\mathrm{VO}_{2}$ máxMe $84 \pm 8 \%$ da FCmáxM no grupo MCF (tab. II). O consumo de oxigênio calculado em $70 \%$ do $\mathrm{VO}_{2}$ máxE foi significantemente maior que o consumo de oxigênio medido no momento em que ocorreu o ponto de compensação respiratória no grupo BCF (fig. 2, A1), mas não no grupo MCF (fig. 2, B1). A prescrição de intensidade de exercício realizada indiretamente em $85 \%$ da FCmáxM não foi estatisticamente diferente da FC medida no ponto de compensação respiratória em nenhum dos dois subgrupos estudados (fig. 2, A2 e B2). No entanto, quando a prescrição de exercício foi realizada em $85 \%$ da FCmáxP, ela foi significantemente maior que a FC medida no ponto de compensação respiratória no grupo BCF (fig. 2, A2) e significantemente maior que a prescrição calculada em $85 \%$ da FCmáxM nos dois grupos estudados (fig. 2, A2 e B2). Esses resultados evidenciam que: 1) o consumo de oxigênio calculado em $70 \%$ do $\mathrm{VO}_{2}$ máxE e preconizado como limite superior de intensidade de exercício para um programa de condicionamento físico foi em média $18 \%$ maior que o consumo de oxigênio medido no ponto de compensação respiratória no subgrupo de $\mathrm{BCF}$, mas não foi diferente no subgrupo de MCF; 2) a FC calculada em $85 \%$ da FCmáxM foi semelhante aquela medida no ponto de compensação respiratória nos subgrupos de BCF e MCF; 3) a FC calculada em $85 \%$ daFCmáxP foi em média $4 \%$ maior que a FC medida no ponto de compensação respiratória no subgrupode BCFe foi em média, 3 e $2 \%$ maior que aFCem $85 \%$ daFCmáxM nos subgrupos BCFe MCF, respectivamente. 


\section{Discussão}

Os principais resultados obtidos no presente estudo foram: 1) o consumo de oxigênio máximo e a FC máxima foram superestimados pelas fórmulas utilizadas em indivíduos de meia idade do sexo masculino; 2) o limite inferior de prescrição indireta de intensidade de exercício para um programa de condicionamento físico calculado em $60 \%$ do $\mathrm{VO}_{2}$ máxE ou em $70 \%$ da FCmáxM foi maior que os valores de consumo de oxigênio e FC medidos no LA; 3) o limite superior de prescrição indireta de intensidade de exercício para um programa de condicionamento físico calculado em $70 \%$ do $\mathrm{VO}_{2}$ máxE ou $85 \%$ daFCmáxM em indivíduos com MCF foi semelhante aos valores de consumo de oxigênio e FC obtidos no ponto de compensação respiratória medidos no teste ergoespirométrico. No entanto, a prescrição indireta calculada em $70 \%$ do $\mathrm{VO}_{2}$ máxM superestima os valores obtidos no teste ergoespirométrico em indivíduos com BCF; para esses indivíduos com BCF apenas a prescrição indireta calculada em $85 \%$ da FCmáxM se assemelha àquela obtida no ponto de compensação respiratória; 4) a prescrição realizada a partir daFCmáxP para a idade fornece limites inferiores e superiores de prescrição de intensidade de exercício físico maiores que os previstos pela FC medida no LA e ponto de compensação respiratória, ampliando os valores já superestimados pela prescrição indireta baseada na FCmáxM.

A discrepância entre os valores do $\mathrm{VO}_{2}$ máx estimado pela fórmula do $\mathrm{ACSM}^{5}$ e aqueles medidos diretamente pela ergoespirometria é provavelmente devida ao tipo de protocolo utilizado durante o TE. A fórmula do ACSM ${ }^{5}$ pressupõe uma condição de equilíbrio (steady-state), enquanto o protocolo de teste utilizado no presente estudo assemelhase ao protocolo de rampa, em que não se permite um equilíbrio, uma vez que os incrementos ocorriam a cada $1 \mathrm{~min}^{7}$. Esses resultados sugerem que na estimativa indireta do $\mathrm{VO}_{2}$ máx deve-se respeitar a fórmula adequada para o protocolo que está sendo empregado ${ }^{8}$. Um outro aspecto a ser considerado, diz respeito à influência da capacidade física nos resultados finais obtidos. No presente estudo observou-se que a discrepância entre os resultados de consumo de oxigênio estimados pela fórmula do $\mathrm{ACSM}^{5}$ foi maior em indivíduos de $\mathrm{BCF}$ que em indivíduos de $\mathrm{MCF}(4 \mathrm{mph}=39 \%$ vs $5 \mathrm{mph}=9 \%$, e BCF $=36 \%$ vs $\mathrm{MCF}=18 \%$ ). Isso pode estar relacionado à cinética de consumo de oxigênio. Estudo anterior do nosso laboratório ${ }^{9}$ mostrou que especialmente em indivíduos mais idosos, um programa de condicionamento físico adequado pode melhorar a relação entre a potência de trabalho e o consumo de oxigênio.

O fato dos indivíduos não atingirem a FCmáxP para a idade durante o TE apesar de todas as avaliações terem sido interrompidas por cansaço físico intenso pode estar relacionado a fadiga muscular. A maior parte dos indivíduos estudados, mesmo os que apresentavam MCF, não estavam acostumados ao exercício em esteira rolante. Assim, é possível que o cansaço físico local possa ter influenciado o momento de interrupção do TE em indivíduos não adaptados ao ergômetro em que esse teste é realizado.
Os limites inferiores de intensidade de treinamento (60\% do $\mathrm{VO}_{2}$ máxE e $70 \%$ da FCmáxM) preconizados pelo $\mathrm{ACSM}^{4}$ para indivíduos saudáveis não idosos foram significantemente maiores que a intensidade relativa do consumo de oxigênio e daFC máxima em que ocorre oLA (46 e $61 \%$, respectivamente). Os valores de $\mathrm{VO}_{2}$ prescritos indiretamente superestimam em aproximadamente $80 \%$ os valores medidos no LA nos grupos de baixa condição física e em cerca de 50\% nos grupos de MCF. Embora essa diferença seja atenuada quando a prescrição é feita, a partir da FCmáxM, ela continua existindo, pois os valores de FC prescritos, indiretamente, superestimam em aproximadamente $14 \%$, os valores de FC medidos no LA, independentemente da capacidade física. Esses resultados demonstram que a prescrição indireta de condicionamento físico aeróbio estimadaem $60 \%$ do $\mathrm{VO}_{2}$ máxE ou 70\% daFCmáxM(limite inferior de intensidade) predispõe o praticante a uma intensidade de exercício em que já ocorre uma intensificação do metabolismo anaeróbio, cuja acidose é compensada pela ativação do sistema tampão e bicarbonato de sódio ${ }^{2}$. Esse fato pode não ter grande importância para indivíduos ativos saudáveis mas, ao contrário, pode ter implicações clínicas para indivíduos sedentários e iniciantes e, principalmente, para portadores de doenças cardiovasculares. Para esses últimos, um estresse metabólico e cardiovascular inadequado, acima dos níveis desejados, pode comprometer os efeitos benéficos causados pelo exercício físico, e em alguns casos, predispor o indivíduo a riscos, como por exemplo, um evento cardiovascular agudo.

Nos indivíduos com menor capacidade física (4mph e $\mathrm{BCF}$ ), o limite superior da prescrição indireta calculada em $70 \%$ do $\mathrm{VO}_{2}$ máxE superestima os valores do consumo de oxigênio medidos no ponto de compensação respiratória, fazendo com que os indivíduos se exercitem numa intensidade em que existe uma acidose metabólica descompensada ${ }^{2}$, limitando a melhora da capacidade aeróbia ${ }^{10}$. Nos grupos com MCF (5mph e MCF), a prescrição com base no $\mathrm{VO}_{2}$ máxE foi semelhante aos valores medidos no ponto de compensação respiratória, isto é, não ultrapassando o limite de uma acidose metabólica compensada. Da mesma forma, a prescrição calculada em $85 \%$ da FCmáxM reflete a FC do ponto de compensação respiratória independentemente da capacidade física.

Um outro aspecto que merece ênfase diz respeito a prescrição baseada na FCmáxP para a idade. Alguns laboratórios têm empregado testes submáximos na avaliação da condição física e, consequientemente, têm prescrito a intensidade de exercício físico para um programa de condicionamento físico, a partir da FCmáxP. A presente investigação demonstra que em todas as condições estudadas, isto é, tanto o limite inferior como o limite superior, independentemente da condição física, a prescrição de intensidade de exercício físico para um programa de condicionamento físico baseada na FCmáxP forneceu valores significantemente maiores que os valores obtidos a partir da FCmáxM. Dessa forma, os erros inerentes da prescrição realizada a partir da FCmáxM foram ampliados pela utilização da FCmáxP. Além 
disso, sabe-se que a FCmáxP apresenta erros que são ampliados à medida que a idade aumenta. Dessa forma, é possível que as distorções apresentadas pelas prescrições indiretas em indivíduos jovens e de meia idade, verificadas no presente estudo, sejam ampliadas em indivíduos idosos, chegando até a invalidar esse tipo de prescrição nessa população. Portanto, é importante que esse estudo seja também realizado em indivíduos idosos.

O presente estudo comparou as prescrições realizadas com base no método direto de ergoespirometria com os métodos indiretos baseados em testes ergométricos padrão. Ele teve por suposição que a prescrição de intensidade de exercício com base no LA ventilatório e no ponto de compensação respiratória seja a mais recomendada para se obter as adaptações benéficas na capacidade cardiopulmonar, verificada após um programa de condicionamento físico ${ }^{2,11,12}$. No entanto, nem o presente estudo, nem outros estudos na literatura compararam o efeito de programas de condicionamento físico prescritos pela forma direta e indireta. Dessa forma, futuros estudos deveriam se ocupar em comparar as adaptações cardiorrespiratórias e metabólicas causadas pelo exercício físico prescrito pelo método indireto (60 - 70\% do $\mathrm{VO}_{2}$ máxE ou 70 - 85\% da FCmáxM) com aquelas causadas pelo exercício físico prescrito pelo método direto através da ergoespirometria (LA e ponto de compensação respiratória).

A fórmula do $\mathrm{ACSM}^{5}$ para cálculo do consumo de oxi- gênio máximo superestima os valores de consumo de oxigênio máximo medidos diretamente durante um teste com incrementos de intensidade a cada $1 \mathrm{~min}$. Indivíduos saudáveis não idosos e não acostumados ao exercício em esteira rolante podem interromper o TE por cansaço muscular antes de atingirem a FCmáxP para a idade. Os limites inferiores de prescrição indireta de intensidade de condicionamento físico aeróbio (60\% do $\mathrm{VO}_{2}$ máxE ou $70 \%$ da FCmáxM) devem ser utilizados com cautela, pois, normalmente, superestimam a intensidade de exercício físico em que o LA é atingido, independentemente da capacidade física, sugerindo que, no início de um programa de condicionamento físico, o limite inferior de prescrição indireta deve ser menor que os valores preconizados pelo $\mathrm{ACSM}^{4}$. O limite superior de uma prescrição de condicionamento físico aeróbio, baseado na FCmáxM, pode ser determinado tanto pelo método de medida direta da análise metabólica quanto pelo método indireto. Por outro lado, o limite superior de prescrição, baseado no $\mathrm{VO}_{2}$ máxE, pode ser usado apenas em indivíduos com MCF. A prescrição de intensidade de exercício baseada na FCmáxP deve ser evitada porque apresenta erros maiores que as demais prescrições indiretas. E, finalmente, para uma precisa prescrição de intensidade de exercício para um programa de condicionamento físico, a capacidade física máxima de indivíduos saudáveis jovens e de meia idade deve-se utilizar a ergoespirometria.

\section{Referências}

1. Individualized Exercise Prescription - In: Noble BJ, eds - Physiology of Exercise and Sport. St. Louis: Mosby, 1986: 263.

2. Skinner JS, McLellan TH - The transition from aerobic to anaerobic metabolism. Res Quaterly Exerc Sport 1980; 51: 234-48.

3. Wasserman K, Hausen JE, Sue DY, Whipp BJ, Casaburi R-Principles of Exercise Testing and Interpretation. $2^{\text {nd }}$ ed. Phyladelphia: Lea \& Febiger, 1994.

4. American College of Sports Medicine - Principles of exercise prescription. In: Guidelines for Exercise Testing and Prescription. $4^{\text {th }}$ ed. Philadelphia: Lea \& Febiger, 1991: 99-100.

5. American College of Sports Medicine-Appendix D: Metabolic Calculations. In: Guidelines for Exercise Testing and Prescription. $4^{\text {th }}$ ed. Philadelphia: Lea \& Febiger, 1991: 96.

6. Miller WC, Wallace JP, Eggert KE- Predicting max HR and the HR-VO relationship for exercise prescription in obesity. Med Sci Sports Exerc 1993; 25: 1077-81.

7. Zhang YY, Johnson MC, Chow N, Wasserman K-Effect of exercise testing proto- col on parameters of aerobic function. Med Sci Sports Exerc 1991; 23: 625-30.

8. Methods. In: Froelicher VF, eds - Manual of Exercise Testing. $2^{\text {nd. }}$ ed. St. Louis: Mosby, 1993: 11 .

9. Wajngarten M, Negrão CE, Kalil LMP et al - Influence of aging and exercise training on the increase in oxygen uptake as a function of the increase in work rate. Cardiol Elderly 1994; 2: 421-6.

10. Training for anaerobic and aerobic power. In: McArdle WD, Katch FI, Katch VL, eds - Exercise Physiology. $3^{\text {rd }}$ ed. Philadelphia: Willians \& Wilkins, 1996: 429-40.

11. Brandão MUP, Wajngarten M, Rondon E, Giorgi MCP, Hironaka F, Negrão CE Left ventricular function during dynamic exercise in untrained and moderately trained subjects. J Appl Physiol 1993; 75: 1989-95.

12. Negrão CE, Forjaz CLM, Rondon MUPB, Brum PC - Adaptação cardiovascular ao treinamento físico dinâmico. In: Souza AGMR, Mansur AJ eds - Cardiologia, Atualização e Reciclagem - SOCESP. 3 ${ }^{a}$ ed. São Paulo: Atheneu, 1996: 532-40. 